Cahiers $d u$ MONDE RUSSE

\section{Cahiers du monde russe}

Russie - Empire russe - Union soviétique et États indépendants

$43 / 4 \mid 2002$

Intellectuels et intelligentsia

\title{
Federico Varese, The Russian mafia
}

\section{Jacques Sapir}

\section{OpenEdition \\ Journals}

Édition électronique

URL : https://journals.openedition.org/monderusse/4043

DOI : $10.4000 /$ monderusse. 4043

ISSN : $1777-5388$

Éditeur

Éditions de l'EHESS

Édition imprimée

Date de publication : 30 décembre 2002

Pagination : 757-763

ISBN : 2-7132-1796-2

ISSN : $1252-6576$

\section{Référence électronique}

Jacques Sapir, «Federico Varese, The Russian mafia », Cahiers du monde russe [En ligne], 43/4 2002, mis en ligne le 17 juin 2009, consulté le 03 septembre 2022. URL : http://

journals.openedition.org/monderusse/4043; DOI : https://doi.org/10.4000/monderusse.4043

Ce document a été généré automatiquement le 3 septembre 2022.

Tous droits réservés 


\title{
Federico Varese, The Russian mafia
}

\author{
Jacques Sapir
}

\section{RÉFÉRENCE}

Federico VARESE, The Russian mafia. Private protection in a new market economy. Oxford, Oxford University Press, 2001, 290 p.

1 L'ouvrage de Federico Varese est l'une des toutes premières études systématiques de la mafia en Russie, qui dépasse avec succès le stade du descriptif, voire simplement de l'anecdotique. C'est un livre important quant à l'économie politique des transactions dans un système faiblement institutionnalisé et l'auteur réussit à rendre un texte dense et fortement structuré théoriquement aussi lisible qu'un bon roman policier.

2 Varese fonde son travail sur l'argument, qu'il emprunte à l'ouvrage de base de Diego Gambetta sur la mafia sicilienne ${ }^{1}$, selon lequel il existerait un lien entre le phénomène mafieux et une offre privée de protection. Celle-ci, dans le cas de la Sicile, provenait de la fin des milices et groupes armés nobiliaires, dont la dissolution correspond à la chute de la monarchie des Bourbons. La demande de protection provient, elle, de la faiblesse de l'État dans sa capacité à vérifier, garantir et protéger les droits de propriété. Varese défend ainsi l'idée d'une distinction nécessaire entre le crime organisé, qui emprunte souvent aux codes comportementaux et opérationnels de la mafia, et la mafia ellemême. Ceci le conduit à la définition suivante : "La mafia diffère du crime organisé en raison de sa relation à l'État. La mafia et l'État sont tous les deux des agences engagées dans la protection. Tandis que la mafia intervient directement dans ce qui relève de la juridiction de l'État, le crime organisé ne le fait pas. De plus, la mafia est disposée à offrir de la protection à la fois à des transactions légales (mais mal protégées) et à des transactions illégales » (p.5).

3 Ceci pourrait conduire à analyser la mafia comme une forme de proto-État. Cependant Varese indique que la différence fondamentale entre la mafia et l'État est que la première ne fonde aucun droit pour les personnes qui recourent à elle, au contraire de l'État. Ainsi doit-on faire une différence entre la mafia et des organisations politico-militaires 
insurrectionnelles qui ont pour vocation de supplanter le pouvoir qu'elles combattent. Cette définition permet alors à l'auteur (p. 6) d'argumenter en faveur d'une comparaison internationale des phénomènes mafieux, tous définis par l'offre de protection comme base de leur activité.

Varese choisit donc comme objet le phénomène de la protection liée à des transactions légales, que celle-ci soit demandée ou imposée, et son terrain est la ville de Perm, choisie à la fois pour sa taille et son éloignement relatif de Moscou. Il élargit ensuite à une recherche concernant les origines du phénomène actuel, portant en particulier sur le rôle des vory-v-zakone (voleurs-sous-la-loi) dont la culture se répand en URSS à partir de 1953, après les libérations de camps. L'auteur a utilisé comme sources tout autant le dépouillement de la presse, les archives que des interviews réalisées lors de séjours de longue durée sur son terrain.

5 Le livre est construit autour de trois parties correspondant à des approfondissements successifs de l'étude de la mafia russe. La première procède à une description du contexte de ces dix dernières années, avec une analyse du processus de transition, une évaluation des capacités de l'État russe comme fournisseur légal de protection et de sécurité, enfin une analyse du spectre général des fournisseurs de protection. Un des résultats les plus intéressants de l'ouvrage est de montrer que les responsables d'entreprises continuent de se pourvoir devant les tribunaux en cas de conflit, même s'ils attendent peu de ces procédures ou s'ils sont conscients qu'il faudra en influencer, sous une forme ou une autre, le déroulement (p. 51-54). En fait, très peu d'acteurs économiques considèrent que le recours à la justice est inutile. Cette situation traduit une réalité où la mafia ne fonctionne pas comme une alternative complète au système légal, mais comme imbriquée dans ce dernier. L'offre privée de protection se manifeste au sein même des canaux de l'offre publique, mais aussi en synergie avec elle. Ceci confirme l'analyse de l'auteur selon laquelle la mafia ne fonctionne pas sur le modèle d'un proto-État mais au contraire dans les interstices de l'État existant. On peut cependant se demander avec l'auteur si cette pénétration interstitielle ne pourrait finir par faire éclater les structures locales et nationales de la puissance publique. Le rôle de groupes locaux, cosaques et divers groupes ethniques, dans la mise en place d'une offre de protection intermédiaire entre l'État et la mafia, pourrait témoigner de ce processus. On peut aussi penser que l'offre publique est susceptible de se reconstituer, mais sous des formes diversifiées. De ce point de vue, la situation semble connaître de fortes variations d'une région à l'autre, en fonction de la plus ou moins grande solidité des autorités locales.

6 Cette analyse du contexte est alors mobilisée dans deux chapitres fondés sur le travail de terrain effectué à Perm : description et analyse des processus de développement de la protection privée à Perm, ainsi que des procédures par lesquelles ce phénomène se développe. On souhaiterait en outre des comparaisons entre régions, justement pour valider (ou invalider) l'hypothèse de l'importance des autorités régionales et des formes de légitimité sur lesquelles elles s'appuient. Double exercice dont on connaît bien la difficulté.

7 La dernière partie du livre est une analyse de fond du phénomène mafieux en Russie, incluant une analyse des antécédents de ce dernier, ainsi que celle de la société des vory$v$-zakone. Ces pages sont certainement parmi les plus intéressantes du texte. La mobilisation des différents ressorts de l'anthropologie est ici particulièrement heureuse.

8 La conclusion que Varese tire de son étude surprend car elle va à l'encontre des lieux communs sur la mafia en général - en tant qu'image d'une société fortement 
structurée et hiérarchisée - et sur la mafia russe en particulier. Il considère ainsi, avec de nombreux éléments à l'appui, que la mafia n'est pas un groupe criminel unifié et surtout qu'elle ne peut pas l'être. Chaque groupe criminel tend à se spécialiser dans une activité (une protection portant sur certains types de transactions) et un territoire. Par contre, à travers la culture des vory-v-zakone, une forme de coordination lâche et informelle se constitue. Le développement de codes fortement ritualisés, d'un discours structuré avec ses mythes, rappelle ici fortement le cas sicilien. Cette culture est avant tout celle des chefs et repose sur un fort élitisme, moyen à la fois de limiter le nombre d'intervenants dans la coordination et de créer une incitation forte dans les rangs des acteurs secondaires. Le rôle des codes opérationnels et sociaux constitue certainement un des points forts du livre, même si on aurait pu souhaiter une plus grande précision des concepts utilisés. Cette partie aurait mérité une comparaison systématique avec l'ouvrage pionnier de Nathan Leites sur le code opérationnel du Bureau politique du PCUS.

9 Cependant, et Varese le montre bien, la mafia russe n'a pas réussi comme la mafia sicilienne. Elle est confrontée à une concurrence sur sa propre activité de base, en particulier à cause de certaines formes de privatisation des organes de sécurité (FSB et MVD), mais aussi en raison du développement d'une logique d'ethnicisation des pratiques criminelles qui favorise une forte fragmentation.

10 Par ailleurs, si la mafia a été utilisée par les grands acteurs politiques et économiques, et si elle a pénétré certains d'entre eux, elle reste plus un instrument à la solde des oligarques qu'un acteur réel dans le monde de l'oligarchie (p.189-191). Ainsi, pour Varese, il est clairement erroné de considérer la Russie comme un « État mafieux » (ce qui serait d'ailleurs dans le cadre de ses concepts une contradiction dans les termes), voire uniquement un État entièrement pénétré et contrôlé par la mafia. De ce point de vue, il rejoint et renforce l'ouvrage de Jeanine Wedel sur l'ascension des oligarques à travers les liens avec l'entourage de El'cin et le soutien de certains groupes d'économistes américains ${ }^{2}$. Les éléments factuels disponibles, en particulier à la suite du scandale qui a impliqué la Bank of New York ou du procès intenté par le gouvernement fédéral contre l'Institut d'aide au développement de l'université de Harvard et son directeur André Shleifer, confirment d'ailleurs amplement cette analyse ${ }^{3}$.

Malgré tous ses mérites, cet ouvrage n'est pas sans limites. Celles-ci tiennent dans une large mesure à l'utilisation d'un appareil théorique qui se situe dans la droite ligne de nombreux travaux sur les institutions mais n'en est pas moins frappé de certaines incohérences.

12 Varese reprend ainsi à son compte un argument qui fut développé par les conseillers américains impliqués dans le processus de privatisation. Les institutions d'une économie de marché ne sauraient ainsi provenir spontanément d'un dictateur bienfaisant ou de parlements éclairés. Elles ne peuvent surgir que des pressions exercées par un groupe social de propriétaires qui, par ces institutions, défendent leurs intérêts ${ }^{4}$. On peut imaginer qu'un législateur ait son ordre du jour rempli par les problèmes découlant des législations et institutions existantes. L'attention qu'il peut accorder à des problèmes nouveaux est bien entendu marginale. Pourtant, cette argumentation contient des incohérences théoriques fortes, mais habituelles dans le cadre de référence utilisé. En effet, le raisonnement tenu par, entre autres, Jonathan Hay et André Shleifer, les deux responsables du Harvard Institute for International Development condamnés par la suite aux États-Unis pour délit de collusion en raison de leur attitude dans le processus de 
privatisation russe $\mathrm{e}^{5}$, revient à dire ceci. Les acteurs, ici les détenteurs de droits de propriété, sont capables d'identifier clairement leurs intérêts à long terme en tant que groupe social, et de les distinguer de leurs intérêts personnels immédiats.

Cette thèse a été régulièrement et systématiquement contredite depuis le début de l'histoire du capitalisme. De la résistance des entrepreneurs britanniques du début du $\mathrm{XIX}^{\mathrm{e}}$ siècle à accepter une limitation du temps de travail et la réglementation du travail des femmes et des enfants, aux pratiques comptables qui ont donné naissance aux scandales Enron et Worldcom, la logique de la prééminence de l'intérêt immédiat et particulier a toujours été la réponse spontanée. Pourtant, il est facile de montrer que des capitalistes qui détruisent la force de travail se condamnent à terme, de la même manière que des capitalistes qui acceptent ou suscitent une manipulation de l'évaluation comptable des droits de propriété. La répétition historique du phénomène renvoie à une question théorique centrale. Supposer, comme les conseillers américains dont Varese reprend l'argumentation, que la vision de l'intérêt de groupe et de long terme puisse spontanément émerger comme représentation dominante revient à supposer que ces agents ne sont pas soumis au principe du « voile d'ignorance ». Mais, si tel était le cas, ils n'auraient pas besoin de droits de propriété car, dans la mesure où ils seraient capables de prévoir le futur dans toutes ses implications, ils seraient en mesure d'établir entre eux des contrats à la fois complets et parfaits. Or, c'est justement l'incapacité d'établir de tels contrats - en raison du voile d'ignorance - qui est une des deux raisons de la nécessité de droits de propriété ${ }^{6}$. L'argument qui tient à la saturation des capacités cognitives du législateur est recevable, mais la solution proposée est, elle, logiquement incohérente et historiquement invalidée.

On peut même aller plus loin. L'argument de Hay, Shleifer et Vishny revient à supposer que le marché puisse spontanément produire les cadres et conditions de son bon fonctionnement. Pourtant, et on le sait depuis plus de 25 ans, ceci est faux dès que l'on admet que l'information dont dispose les agents n'est ni complète ni parfaite ${ }^{7}$. L'un des trois Prix Nobel d'économie de 2001, Joseph Stiglitz, en avait d'ailleurs tiré les conséquences quant au rôle de l'État dans la mise en place des institutions ${ }^{8}$. Federico Varese cite les travaux de Stiglitz réalisés quand il était l'économiste en chef de la Banque mondiale; pourtant, il n'en tire pas les conséquences logiques du point de vue théorique.

15 L'argumentaire théorique de Varese soulève aussi d'autres problèmes. Les échecs dans la mise en œuvre d'une réglementation adéquate sont pour lui assimilés au problème de la relation Principal/Agent, un des instruments développés justement par les économistes qui se sont penchés sur les asymétries d'information'. Une approche par la légitimité aurait été plus cohérente avec la démarche anthropologique adoptée à propos de l'influence de vory-v-zakone sur le développement de la mafia russe. Ceci impliquerait cependant une analyse des divers types de légitimité, et l'absence de Max Weber dans les références est ici une limite claire de l'appareil conceptuel que l'auteur a mobilisé dans son étude ${ }^{10}$.

16 Prendre au sérieux la question de la légitimité dans l'explication des crises des institutions impliquerait alors de réintroduire la politique économique en amont du problème de la demande de protection. Comment en effet imaginer que des policiers et des juges puissent raisonnablement faire leur travail s'ils sont mal payés, voire laissés sans solde. Comment imaginer qu'un système juridique puisse être perçu comme légitime quand les jugements sont erratiques, les procédures longues et incertaines, et 
quand globalement le fonctionnement du système social est en contradiction avec ses propres valeurs. De ce point de vue, on ne peut dissocier le développement de la mafia, au sens où Varese l'entend, des effets de la politique économique mise en place entre 1992 et 1998, mais aussi du comportement du pouvoir eltsinien vis-à-vis des institutions, comme l'a tragiquement démontré l'affaire de la dissolution du Parlement de Russie en octobre 1993. Si le développement des pratiques mafieuses constitue bien une contrainte sur les possibilités d'action future, ce développement est aussi le produit - bien entendu non intentionnel - des actions passées.

17 Un dernier point qui mérite discussion est celui du lien entre les institutions d'une économie de marché et les droits de propriété au premier chef, et l'État. Varese indique à plusieurs reprises l'existence de continuités entre l'URSS et la Russie post-soviétique.La question du sous-développement de l'État dans le système soviétique est à l'évidence indissociable des formes prises par la criminalité économique. Or les protagonistes de la transition n'ont jamais eu, sauf à de très rares exceptions, la conscience que la sortie du soviétisme passait par la reconstruction de l'État. Bien au contraire, le préjugé antiétatiste a été dominant pendant la période la plus cruciale, 1989-1996. L'idéologie de la dévalorisation de l'État a joué un rôle considérable dans l'affaiblissement des institutions publiques durant le début de la transition. Ce phénomène a été renforcé par la rapide diminution des moyens financiers dont les autorités publiques pouvaient disposer, en particulier à cause des politiques budgétaires mises en place sur les conseils des experts américains et du FMI.

Ceci n'implique pas qu'il ne fallait pas engager un processus de privatisation dans une économie où l'essentiel de la production était étatisé. Cependant, il était d'emblée dangereux de présenter la privatisation comme une solution miracle. La mise en place de ce processus aurait dû être plus lente et plus prudente. L'absence d'une discussion sur les effets pervers de l'idéologie de la privatisation, à distinguer de pratiques qui peuvent être nécessaires, sur l'autorité de l'État et sur sa légitimité, est d'autant plus à regretter que, fort justement, Varese analyse le développement de la mafia en conjonction avec une faillite de l'État.

Plus loin encore, il faudrait s'interroger sur le statut des droits de propriété. Si la combinaison de la densité sociale et du voile d'ignorance rend nécessaires des droits de propriété individuels, cette même combinaison, quand elle concerne les effets de l'usage de ces droits, rend impérative l'existence de droits collectifs, qui se manifestent entre autres sous la forme des réglementations publiques concernant l'usage des biens et services appropriés par les individus. Il n'est donc pas possible de penser une propriété privée "pure ", au sens de dégagée de toute obligation collective, car les conditions d'existence d'une telle propriété seraient celles rendant l'existence même des droits de propriété superflue ${ }^{11}$. Cette nécessaire combinaison de l'individuel et du collectif a des conséquences quant à la définition, la vérification et la protection des droits de propriété mobilisés dans des transactions. Elle rend illusoire toute approche opposant les sphères privée et publique.

20 Au-delà de ces critiques qui visent moins Varese directement que le courant dans lequel il se situe, il est important de redire que son ouvrage est la première étude sérieuse et systématique du phénomène mafieux dans la Russie post-soviétique. La combinaison des approches théoriques utilisées fournit des pistes de recherche qui devraient s'avérer très fructueuses dans les années à venir. Il constitue à l'évidence un outil indispensable à la 
fois pour le chercheur qui travaille sur la Russie et la transition, mais, au-delà, pour qui s'interroge sur le phénomène du crime économique et de la mafia.

\section{NOTES}

1. Diego Gambetta, The Sicilian mafia, Cambridge, MA, Harvard University Press, 1993.

2. J. Wedel, Collision and collusion, New York, St. Martin's Press, 1998.

3. J. Sapir, Les économistes contre la démocratie, Paris, Albin Michel, 2002 (chapitre 1).

4. J. R. Hay, A. Shleifer et R. W. Vishny, « Toward a theory of legal reform ", European Economic Review, 40 (3-5), 1997, p. 559-567, voir p. 564.

5. Sur le comportement de Shleifer en particulier, voir M. Bivens, "Harvard's "fitting choice" ", édition électronique du Moscow Times, lundi 18 juin 2001.

6. L'autre étant l'existence d'une densité sociale. Voir J. Sapir, Les trous noirs de la science économique, Paris, Albin Michel, 2000.

7. Voir, par exemple, S. J. Grossman, J. Stiglitz, "Information and competitive price systems ", American Economic Review, 66 (3), mai 1976, Papers and Proceedings of the Annual Meeting of the American Economic Association.

8. J. Stiglitz, "Wither reform? Ten years of transition », Keynote Address to the Annual Bank Conference on Development Economics, World Bank, Washington DC, 28-30 avril 1999.

9. Pour son origine, voir S. Ross, "The economic theory of the agency: the principal's problem ", American Economic Review, 63, (1), mars 1973, p. 134-139. Voir aussi, B. Holmstrom, "Moral hazard in teams", Bell Journal of Economics, 13, 1982, p. 324-340. Pour une bonne présentation pédagogique, J.J. Laffont, Economie de l'incertain et de l'information, Paris, Economica, 1991, chapitres 4, 5 et 11.

10. Voir Max Weber, The theory of social and economic organization, Londres - New York, The Free Press - Macmillan, 1964 (traduction de la partie I de Wirtschaft und Gesellschaft) et en particulier la troisième partie du volume.

11. Sur ce point, voir J. Sapir, Les trous noirs de la science économique, op. cit. 